ScIDoc
International Journal of Dentistry and Oral Science (IJDOS)

ISSN: $2377-8075$

\title{
Obstructive Sleep Apnea In Children - A Review
}

Prathap Chandar Manivannan ${ }^{1}$, Swarna Swathi Silla ${ }^{2 *}$, Sarada Penmetcha ${ }^{3}$

${ }^{1}$ Associate Professor, Department of Orthodontics, Faculty of Dentistry, MAHSA University, Selangor, Malaysia.

${ }^{2}$ Associate Professor, Department of Pedodontics and Preventive Dentistry, Sri Sai College of Dental Surgery, Vikarabad, Telangana, India.

${ }^{3}$ Professor, Department of Pedodontics and Preventive Dentistry, Sri Sai College of Dental Surgery, Vikarabad, Telangana, India.

\section{Abstract}

\begin{abstract}
Obstructive sleep apnea (OSA) is a sleep-related breathing disorder characterized by episodes of upper airway collapse during sleep. OSA is one of the most common causes of sleep disordered breathing in children. Pediatric OSA affects 2 to $5 \%$ of school-aged children. The pathophysiology of this disorder in children is multifactorial, but two significant risk factors include adenotonsillar hypertrophy and obesity. Snoring, difficulty in breathing and tiredness are prominent symptoms in children. Pediatric OSA has been linked to metabolic changes, growth inhibition, and cardiovascular sequelae. Consequences of untreated OSA include failure to thrive, enuresis, attention deficit disorder, behavior problems, poor academic performance and cardio pulmonary disease.
\end{abstract}

Keywords: Obstructive Sleep Apnea (OSA); Adenotonsillar Hypertrophy (AT); Polysomnography (PSG); Continuous Positive Airway Pressure (CPAP).

\section{Introduction}

Sleep is a universal phenomenon that is characterized by a reversible state of partial unresponsiveness and disengagement from the environmentand amajor physiological drive. It is critical to child's health, development and daily functioning [1]. During sleep, several physiological processes occur like fall in body temperature, increased sleep glucose metabolism, decrease durine, sodium andpotassium excretion and decreased secretion of cortisol and TSH [2].

Various sleep disorders include in somnia, sleep related breathing disorders, narcolepsy, circadian rhythm disorder, parasomnias and sleep related movement disorders. Apnea is the cessation of air flow for at least 10 seconds with drop in oxygen saturation, whereassleepapnea is intermittent cessation of airflow in nose and mouth during sleep [3].

Obstructive Sleep Apnea (OSA) is a condition in which there is repetitive partial or complete collapse of the pharynx during sleep. OSA associated with excessive daytime sleepiness is called Obstructive Sleep Apnea Syndrome (OSAS) [4]. OSAS is the co- existence of unexplained excessive day time sleepiness with at least five obstructed breathing events per hour of sleep. According to American thoracic society OSAS is a disorder of breathing during sleep characterized by prolonged partial upper airway obstruction and/or intermittent complete obstruction [5]. OSAS is a significant cause of morbidity and mortality throughout the world and the most common medical cause of daytime sleepiness [6].

Charles Dickens was the first person who provided the comprehensive description of what, about 150 years later came to be known as obstructive sleep apnea [6]. In 1918, William Osler described obstructive sleep apnea syndrome as sleep related disordered breathing. Recently, the term "complex sleep apnea syndrome" has been introduced which has gained popularity. Obstructive sleep apnea (OSA) is one of the major sleep disorders of childhood [7].

\section{Epidemiology}

Pediatric obstructive sleep apnea has been widely recognized only in last few decades as a likely cause of significant morbidity among children [8]. In prepubertal children, the incidence of

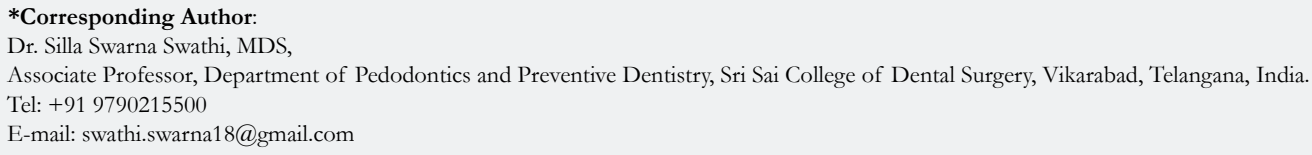

Copyright: Swarna Swathi Silla 2021 . This is an open-access article distributed under the terms of the Creative Commons Attribution License, which permits unrestricted use, distribution and reproduction in any medium, provided the original author and source are credited. 
OSAS is similar in boys and girls, after puberty it is more common in boys than girls [9]. OSAS prevalence has two peak periods. The first peak occurs in children between 2 to 8 years of age with the presence of enlarged adenoids and or tonsils. Second peak arises during adolescence in relation with weight gain. Boys are more affected than girls [10]. Childhood OSAS is an increasingly recognized morbidity affecting $2-5 \%$ of children specifically Asian Indian children due to growing urbanization and nutrition transition, obesity and metabolic syndrome [11].

\section{Etiology and Pathogenesis}

Etiology for pediatric OSA includes:

- Obesity

- Adenotonsillar hypertrophy

- Mandibular deficiency

- Macroglossia

- Upper airway tumors(rare)

- Loss of muscletone

- Obstruction of nasalpassages

- Micrognathia

- Macroglossia

- Retrognathia.

Some genetic syndromes such as Pierre Robin syndrome, Down syndrome, hypothyroidism, Arnold Chiari malformation, myotonic dystrophy and other neuromuscular disorders may be associated with OSAS [12].

The pathogenesis of pediatric OSA is complex and involves a complex interplay between functional changes which occur normally during sleep and anatomical factors both which lead to the narrowing of upper airway and subsequently to partial or complete obstruction [13]. OSA occurs as a consequence of an anatomical reduction in the upper airway or in coordination of upper airway dilatory muscle activity [14].

The pathophysiology of pediatric obstructive sleep apnea can be divided into factors that affect upper airway collapsibility, factors that produce anatomic narrowing or a combination of both. The factors that produce anatomical narrowing include adenotonsillar hypertrophy (AT), allergicrhinitis, turbinate hypertrophy, deviated septum and maxillary constriction. Among these adenotonsillarhypertrophy is considered to be the most common cause of OSAS in children. Airway narrowing can also be due to craniofacial abnormalities and/or soft tissue hypertrophy. An event of airway narrowing includes obstructive cycling, increased respiratory effort, flow limitation, tachypnea, and/or gas exchange abnormalities. Consequently sleep disruption occurs, ranging from visible electrocortical arousal to subtle autonomic activation [15].

Other contributors to anatomical obstruction include macroglossia, increased nasal resistance, craniofacial anomalies (retrognathia, micrognathia, and midface hypoplasia) and lingual tonsil hypertrophy. Lingual tonsil hypertrophy can obliterate the vallecula, pushing the epiglottis posteriorly, resulting in significant obstruction. Lingual tonsil hypertrophy is another contributor and an important cause of residual OSAS after adeno tonsillar hypertrophy [9].
Adenotonsillar hypertrophy has also been noted to be associated with increased upper airway collapsibility. It affects $2 \%$ of normal children aged between 2 to 8 years and may lead to significant neurocognitive deficits and cardiovascular dysfunction if untreated [16]. The most common site of obstruction in adenotonsillar hypertrophy is in retro palatal region. Once obstruction occurs, impaired ventilation leads to hypoxia and hypercapnia with subsequent increased respiratory effort and finally arousal from sleep in order to re- establish airway patency. This cycle repeats several times throughout the night, resulting in recurrent hypoxia and fragmentation of sleep [15].

While previously, the typical pediatric OSA patient was one with adenotonsillarhypertrophy, with the current obesity endemic, there are an increasing number of children diagnosed with OSA who are obese [17]. Obesitycontributestoreductioninupperairwaysizebyincreasingtheamount of fat deposited in the soft tissues of the pharynx or by compressing the pharynx by superficial fat masses in theneck. Obesity is also one of the major causes of OSA [18].

\section{Clinical Signs}

Clinical signs of OSA include

- Continuoussnoring

- Pauses in breathing while a sleep

- Indifferent legposition

- Enlarged tonsils

- Stunted growth and disruptive behavior inschool

- Frequent snoring

- Excessive daytime sleepiness

- Restlesssleep

- Paradoxical chest movement

- Parasomnias

- Nocturnalarousals

- Cyanosis

- Bedwetting hyperactivity

- Stunted growth and disruptive behavior inschool

- Excessive daytime sleepiness and snoring with pauses is usually seen in adults [19].

These symptoms will vary with age.

- Infants: Child of 3-12 months (infants) presents less common symptoms. Noisy breathing rather than snoring is a usual complaint of the parents of the infant .

- Toddlers: Snoring becomes more common at this age (2 to 5 years) when the tonsils and adenoids are thought to relatively narrow the airway. Children at this age present with sleep terrors or confusional arousals, as well as moving around the bed in restless sleep in order to maintain their airway. Children at this age rarely complain of daytime sleepiness and more often will be hyperactive' and irritable and also have insomnia.

- Pre-school: This age group tends to present symptoms in a similar fashion to toddlers, but drooling may become more apparent and nocturnal enuresis becomes an issue. Enuresis alone at this age is not a concerning symptom as it occurs in up to $15 \%$ of normal children. As children start kindergarten and school and need to wake up at the same time each morning, waking the child due to improper sleep pattern become more and more difficult. 
In this age group, children are also able to communicate symptoms and may start to complain of headaches on awakening in the morning. Sleeping in the knee-chest position at this age is abnormal and indicative of airwaycollapsibility.

- School age: These children have symptoms of hyperactivity and inattention and may be labelled with Attention Deficit Hyperactivity Disorder (ADHD). As permanent teeth starts erupting, malocclusion may become more apparent [20].

The three main night time symptoms of OSA in infants and children are snoring, apnea with noisy resumption of breathing and difficulty in breathing with an inward movement of the upper chest during inspiration. In addition to snoring; the majority of children with sleep disordered breathing who are referred to otolaryngologists have mouth breathing and adenotonsillar hypertrophy. The relationship between mouth breathing and adenoidal hypertrophy is straight forward. Mouth breathing is a significant predictor for suspecting OSA with a specificity and positive predict value of $100 \%$ and warrants early polysomnography [19]. Most children with OSA breathe normally while awake and have minimal day time symptoms. Excessive daytime sleepiness, the most prominent clinical symptom of OSA in adults, is not a common complaint in pediatric sleep disordered breathing. However, it is seen in some children with severe OSA. Younger children often become hyperactive rather than sleepy [22].

\section{Orofacial Findings}

Common orofacial characteristics of obstructive sleep apnea patients are:

- Retrognathic mandible

- Narrow palate

- large neck circumference

- Long soft palate

- Tonsillar hypertrophy

- Nasal septal deviation

- Relative macroglossia [16].

\section{Risk factors of OSA in children}

A child may have morethan one risk factor for OSA. The relationship between risk factors and the probability of having OSA is directly proportional. More the risk factors a child has, greater the chance of having sleepapnea.

Risk factors of OSA in children [15].

\section{Diagnosis of OSA}

Diagnosis of OSAS is based on history, clinical suspicion and physical findings. Confirmation is made by polysomnography (PSG).

History and Examination: A thorough history should be taken including detailed information on nighttime and daytime symptoms as well as OSAS associated morbidities such as neurobehavioral deficits, behavior, sleepiness, failure to thrive and systemic hypertension [22]. History of snoring during night is most significant in predicting OSA [23].

Attention should be directed to the size of the tonsils and their position, the presence of allergic rhinitis or any other condition which are likely to increase nasal airflow resistance and the relative size (micrognathia) and positioning (retrognathia) of the mandible [24].

A comprehensive physical examination of the upper airway from the nose to the oropharynx can help to find any anatomical narrowing and it includes the following successive segments.

1. The nose should be examined for asymmetry of then ares, a large septal base, collapse of the nasal valves during inspiration, a deviated septumore $\mathrm{nl}$ argement of the inferior nasal turbinates. 2. The oropharynx should be examined for the position of the uvula in relation to the tongue.

3. The size of the tonsils should be compared with the size of theairway.

4. The presence of a high and narrow hard palate, overlapping incisors, a crossbite and a $2 \mathrm{~mm}$ of over jet are indicative of a small jaw and/or abnormal maxilla-mandibular development [25].

Clinical history and physical examination cannot accurately discriminate between habitual snorers and patients with obstructive sleep apnea. The American Academy of Pediatrics concluded that the positive predictive value of the clinical history was $65 \%$ and the positive predictive value of the physical examination was $45 \%$ and recommended objective testing with overnight polysomnography [9]. Other diagnostic factors for OSA are neck circumference and the presence of fatty infiltration [25].

Anadenoid face with mouth breathing at the waking state is an important clue in detecting sleep-disordered breathing. In spection of the lateral facial profile is helpful to evaluate for retrognathia,

Table 1.

\begin{tabular}{|c|c|c|}
\hline ANATOMIC & $\begin{array}{c}\text { ANAMOLIES OF } \\
\text { CRANIOFACIAL BONES }\end{array}$ & FUNCTIONAL \\
\hline \multirow{2}{*}{ - Nasalpolyps } & \multirow{2}{*}{ - Retrognathia } & - Generalized hypotonia in neuromuscular diseases \\
\hline & & - $\quad$ E.G muscular dystrophies \\
\hline - $\quad$ Allergicrhinitis & - $\quad$ Micrognathia & - $\quad$ Effect ofsedative hypnotics \\
\hline \multirow[t]{4}{*}{ - $\quad$ Nasaltumors } & - $\quad$ Midfacehypoplasia & - $\quad$ Achondroplasia \\
\hline & - $\quad$ Cleftpalate & - $\quad$ Spina bifidamyelomeningocele \\
\hline & - $\quad$ Macroglossia & - $\quad$ Mucopolysacharidosis \\
\hline & - Obesity & \\
\hline
\end{tabular}


micrognathia or midfacial hypoplasia [19].

In advanced cases of OSAS, a loud second pulmonary heart sound may manifest as an evidence of pulmonary hypertension. Assessment for growth and development should not be omitted because growth impairment and delayed development are frequently associated in children with OSAS. Para nasal sinus with neck lateral view in X-ray is a simple but very useful method for the detection of sinusitis or adenoid hypertrophy. Endoscopy performed under sedation is useful in localizing the region of maximal airway restriction. This technique is reserved for children with complicated airway structure and altered collapsibility such as congenital craniofacial anomaly [19].

Polysomnography: Polysomnography (PSG) is the Gold Standard for the Diagnosis of OSAS and Severity Assessment.PSG include electroencephalogram (EEG) leads, electrooculogram (EOG), electromyogram (EMG), nasal pressure/oral thermistor, electrocardiogram (ECG), pulse oximetry, chest and abdominal excursion belts, plethysmography, limbleads, endtidalor transcutaneous $\mathrm{CO}_{2}$, oesophageal manometry and audio/video taping. The information provided from these parameters can evaluate the sleep architecture, breathing events during sleep (including apneas, hypopneas, flow limitation, respiratory effort related arousals), desaturation and periodic limb movement as well as autonomic changes and respiratoryeffort [25].

Further diagnostic methods such as audio taping, videotaping, questionnaires, home monitoring device, overnight pulse oximetry, tracheal sound signals or sleep endoscopy have been established. In the future, polygraphy, urinary biomarkers and rhinomanometry, all show high diagnostic test accuracy, might be alternatives to PSG [26].

\section{Consequences}

Understanding sleep disorders in general, and more particularly, sleep-disordered breathing, can lead to substantial morbidities affecting the central nervous system (CNS), the cardiovascular and metabolic system and somatic growth, ultimately leading to reduced quality oflife [27].

\section{Complications}

1.Behavioral:

- Aggression

- Hyperactivity

- Anxiety

- Depressed mood

- Psychosocial difficulties

- Nocturnalenuresis

2. Cognitive

- Inattentiveness

- Impaired executive functioning

- Impaired memory

- Impaired scholastic function

3. Cardiopulmonary
- Systemic hypertension

- Impaired right ventricular function-rare

- Cor pulmonale -rare

4. Growth andmetabolism

- Failure tothrive

- Delayed physical growth [28].

\section{Treatment}

Obstructive sleep apnea can be managed via surgical or non surgical methods. Treatment modality for OSA depends on the following:

1) Severity of the patient'ssymptoms.

2) Results of the polysomnogram.

3) Impact on co-morbid diseases such as heart failure [16].

The surgical methods include:

- Adenotonsillectomy (most common inchildren)

- Rapid maxillary expansion

- Maxillo-mandibular advancement

- Uvulopalatopharyngoplasty

- Craniofacial surgeries

- Cleft palate revisionprocedures [16].

Nonsurgical methods for the treatment of obstructive sleep apnea include:

- Continuous positive airway pressure (CPAP)

- Diet

- Medications

- Oralappliances [16]

\section{Sugrical Methods}

Adenotonsillectomy: Tonsillectomy is a surgical procedure where the peri-tonsillar space between the tonsillar capsule and muscular wall is dissected and the tonsil is completely removed. When performed in conjunction with removal of the adenoids, this procedure is referred to as adenotonsillectomy. If a child has OSAS and has adeno tonsillar hypertrophy, adenotonsillectomy is considered as the first line therapy [12].

Rapid Maxilliary Expansion (RME): RME is an orthodontic procedure that uses a fixed appliance with an expansion screw anchored on molar teeth, which can be helpful if the child has a deviated septumor narrow nasal passages. The principal goal of RME in children with a reduced nasopharyngeal and oropharyngeal airway is to address the posterior crossbite and widen the maxilla. The expansion occurs within three weeks and the device remains in situ for three months to allow for remodeling of the cartilage [29].

Maxillomandibular Advancement: Maxillomandibular advancement is a very successful procedure. Nonetheless, it is a major surgery that should be performed after there has been apt orthodontic treatment.It may be performed at any time during 
childhood, but it is often postponed until 11 to 12 years of age. [30].

Uvulopalaptopharyngoplasty (UPPP): Surgery of the oropharynx for the management of OSA is Uvulopalatopharyngoplasty. Fujita et al (1981) first described the use of UPPP for the treatment of OSA. Uvulopalatopharyngoplasty, comprises expansion of the oropharyngeal airwayby removal of tonsillar tissue on the anterior surface of the soft palate, as well as the uvula. This is a common procedure done for management of adult OSA. Uvulopalatopharyngoplasty in combination with adenotonsillectomy was described as an alternative to tracheostomy in children with significant neurological impairment and moderate to severe OSA [31].

Tracheostomy: Tracheostomy offers complete relief of upper airway obstruction and facilitates accessto the lower airway for pulmonary as well as mechanical ventilation [32].

Nasal Treatment: Buryand Singh(2015) highlightedthatnasalsurgeryforobstructivesleepapneapatients increase the quality of life and treatment compliance in some affected patients [33].

Lingual Tonsillectomy: In persistent OSAS severe enough to warrant additional surgery, lingual tonsillectomy can be performed using a number of different techniques, including radio frequency ablation, laser, microdebrider, and suction electrocautery [34].

\section{Non Surgical Management of OSA}

Diet and Medications: In overweight children, weight control and maintaining healthy diet is an important treatment modality. In children who are still growing, weight maintenance may be more appropriate than weight loss as the child will continue to increase height [35].

Mild persistent OSA after adenotonsillectomy may be successfully treated with nasal corticosteroids and oral anti- inflammatory therapy such as leukotriene inhibitors e.g. montelukast [36].

Continuous Positive Air Pressure (CPAP): Continuous positive airway pressure (CPAP) is a treatment option for children, in managing moderate to severe Obstructive Sleep Apnea cases and also for the treatment of children whose Obstructive Sleep Apnea symptoms are not relieved after adenotonsillectomy.

CPAP is delivered using an electronic device that delivers constant air pressure via nasal mask, leading to mechanical stunting of the airway and improved functional residual capacity in the lungs. CPAP in treatment of children involves wearing a mask overnight that exerts pressure by acting as a pneumatic splint on the upper airway to prevent collapse [37].

Oral Appliances (OAs): The use of oral appliances has involved dentists in the treatment of Obstructive Sleep Apnea among both adults and children. The role of oral appliances (OAs) in the treatment of patients with OSA has gained prominence since their introduction in 1982. Recently, the American Academy of Sleep Medicine published practice parameters for the treatment of snoring and OSA withOA establishing a first-line role for OA in the treatment of adult patients with mild-to moderate OSA, and a second-line role for patients with severe OSA [37].
Kushida C.A. et al. (2006) reported that mandibular advancement appliances were capable of reducing snoring and mild to moderate Obstructive Sleep Apnea in about 30\% to 54\% of patients [38].

Currently, over 40 different types of oral appliances are available to treat OSA Syndrome. These devices are worn only during sleep, which advances the mandible or tongue, increasing the size of the upper airway. Oral appliances have been used in adults but have concerns with altering the bite inchildren which prevents its use. The main indication for the use of oral appliances in obstructive sleep apnea cases is patients who choose to have neither surgery nor continuous positive airway pressure [37].

American Academy of Pediatrics guidelines for the diagnosis and management of obstructive sleep apnea syndrome (OSAS):[39]

(1) All children/adolescents should be screened for snoring. (2) Polysomnography should be performed in children/adolescents with snoring and symptoms/signs of OSAS; if polysomnography is not available, then alternative diagnostic tests or referral to a specialist for more extensive evaluation may be considered. (3) Adenotonsillectomy is recommended as the first-line treatment of patients with adenotonsillar hypertrophy. (4) High-risk patients should be monitored as inpatients postoperatively. (5) Patients should be re-evaluated postoperatively to determine whether further treatment is required. Objective testing should be performed in patients who are high risk or have persistent symptoms/signs of OSAS after therapy. (6) Continuous positive airway pressure is recommended as treatment if adenotonsillectomy is not performed or if OSAS persists postoperatively. (7) Weight loss is recommended in addition to other therapy in patients who are overweight or obese. (8) Intranasal corticosteroids are an option for children with mild OSAS in whom adenotonsillectomy is contraindicated or for mild postoperative OSAS.

\section{Conclusion}

In the recent decades since the initial description of OSA in children, have witnessed extensive and meaningful progress in research on the etiologic factors, pathophysiology, diagnosis and treatment of pediatric OSA. A greater understanding of the mechanisms underlying the pathogenesis of the disease will be required for proper management of children and to prevent adverse consequences.

\section{References}

[1]. Karen J Marcdante and Robert M Kliegman. Normal sleep and pediatric sleep disorders. Nelson Essentials of Pediatrics, 7thed. Elsevier Publications 2014;p: 47-50

[2]. Fauci,Brauwald E, Kasper, Hauser, Longo, Jameson and Loscalzo. Harrison's Textbook of internalmedicine. Mc Graw Hill Education; 17th ed 2008.

[3]. Ionescu Clara Mihaela. The human respiratory system. Springer Publications,London; 2013: p 13-22.

[4]. Zhang W, Si LY. Obstructive sleep apnea syndrome (OSAS) and hypertension: pathogenic mechanisms and possible therapeutic approaches. Ups J Med Sci. 2012 Nov;117(4):370-82.Pubmed PMID: 23009224.

[5]. Arali V, Namineni S, Sampath Ch. Pediatric obstructive sleep apnea syndrome: time to wake up. Int J Clin Pediatr Dent. 2012 Jan;5(1):54-60.Pubmed PMID: 25206135.

[6]. GuptaRK,ChandraA,VermAK,KumarS.Obstructivesleepapnea:Aclinicalrevi 
ew. J Assoc Physicians India. 2010; 58:438-41

[7]. Nagarajappa AK, Dwivedi N. Sleep apnea: A dental perspective. J Indian Acad Oral Med Radiol. 2015 Apr 1;27(2):223-9.

[8]. Lumeng JC, Chervin RD. Epidemiology of pediatric obstructive sleep apnea. Proc Am Thorac Soc. 2008 Feb 15;5(2):242-52.

[9]. DelRosso LM. Epidemiology and diagnosis of pediatric obstructive sleep apnea. Curr Probl Pediatr Adolesc Health Care. 2016 Jan;46(1):2-6.

[10]. Moon IJ, Han DH, Kim JW, Rhee CS, Sung MW, Park JW, et al. Sleep magnetic resonance imaging as a new diagnostic method in obstructive sleep apnea syndrome. Laryngoscope. 2010 Dec;120(12):2546-54.

[11]. Marcus CL, Keens TG, Bautista DB, von Pechmann WS, Ward SL. Obstructive sleep apnea in children with Down syndrome. Pediatrics. $1991 \mathrm{Jul}$ $1 ; 88(1): 132-9$

[12]. Tauman R, Gozal D. Obstructive sleep apnea syndrome in children. Expert Rev. Respir.Med. 2011;5(3):425-40.

[13]. Tanné F, Gagnadoux F, Chazouilleres O, Fleury B, Wendum D, Lasnier E, et al. Chronic liver injury during obstructive sleep apnea. Hepatology. 2005 Jun;41(6):1290-6.

[14]. WarwickJP and Mason. Obstructive sleep apnea in children. Anesthesia. 1998; 53: 571-579.

[15]. Chhangani BS, Melgar T, Patel D. Pediatric obstructive sleep apnea. Indian JPediatr. 2010;77(1):81-5.

[16]. Jaradat M, Rahhal A. Obstructive Sleep Apnea, Prevalence, Etiology \& Role of Dentist \& Oral Appliances in Treatment. OJST. 2015;5(07):187-201.

[17]. Dehlink E, Tan HL. Update on paediatric obstructive sleep apnoea. J Thorac Dis. 2016 Feb;8(2):224-235.

[18]. Muzumdar H, Arens R. Physiological effects of obstructive sleep apnea syndrome in childhood. Resp Physiol Neurobi. 2013 Sep 15;188(3):370-82.

[19]. Chang SJ, Chae KY. Obstructive sleep apnea syndrome in children: Epidemiology, pathophysiology, diagnosis and sequelae. Korean J. Pediatr. 2010 Oct;53(10):863-71.

[20]. Sinha D, Guilleminault C. Sleep disordered breathing in children. Indian J Med Res. 2010 Feb 1;131(2):311-20.

[21]. Tangugsorn V, Skatvedt O, Krogstad O, Lyberg T. Obstructive sleep apnoea: a cephalometric study. Part I. Cervico-craniofacial skeletal morphology. Eur J Orthod. 1995 Feb;17(1):45-56.Pubmed PMID: 7737345.

[22]. Thorpy MJ. Classification of sleep disorders. Neurotherapeutics. 2012 Oct;9(4):687-701.

[23]. Dayyat E, Kheirandish-Gozal L, Gozal D. Childhood obstructive sleep apnea: one or two distinct disease entities?. Sleep Med. Clin. 2007 Sep $1 ; 2(3): 433-44$.

[24]. Tauman R, Gozal D. Obstructive sleep apnea syndrome in children. Expert Rev Respir Med. 2011 Jun 1;5(3):425-40.

[25]. Huynh NT, Desplats E, Almeida FR. Orthodontics treatments for managing obstructive sleep apnea syndrome in children: A systematic review and metaanalysis. Sleep Med Rev. 2016 Feb 1;25:84-94.

[26]. Ciccone DK, Vian T, Maurer L, Bradley EH. Linking governance mecha- nisms to health outcomes: a review of the literature in low-and middle-income countries. Soc Sci Med. 2014 Sep 1;117:86-95.

[27]. Linder-Aronson S, Woodside DG, Lundström A. Mandibular growth direction following adenoidectomy. Am J Orthod. 1986 Apr;89(4):273-84. Pubmed PMID: 3515955.

[28]. Peltomäki T. The effect of mode of breathing on craniofacial growth--revisited. Eur J Orthod. 2007 Oct;29(5):426-9.Pubmed PMID: 17804427.

[29]. Imanguli M, Ulualp SO. Risk factors for residual obstructive sleep apnea after adenotonsillectomy in children. Laryngoscope. 2016 Nov; 126(11):2624-

[30]. Guilleminault C, Li KK. Maxillomandibular expansion for the treatment of sleep-disordered breathing: preliminary result. Laryngoscope. 2004 May;114(5):893-6.Pubmed PMID: 15126751.

[31]. Isaiah A, Mitchell RB. Pediatric Obstructive Sleep Apnea: Surgical Techniques beyond Tonsillectomy and Adenoidectomy. Int J Head Neck Surg 2016;7(2):109-114.

[32]. Maturo SC, Mair EA. Submucosal minimally invasive lingual excision: an effective, novel surgery for pediatric tongue base reduction. Ann Otol Rhinol Laryngol. 2006 Aug;115(8):624-30.Pubmed PMID: 16944662.

[33]. Kumar AR, Guilleminault C, Certal V, Li D, Capasso R, Camacho M. Nasopharyngeal airway stenting devices for obstructive sleep apnoea: a systematic review and meta-analysis. J Laryngol Otol. 2015 Jan;129(1):2-10.Pubmed PMID: 25544266.

[34]. Badełek-Izdebska M, Dębska M, Zawadzka-Głos L. LinguaL tonsiL hypertrophy as a cause of obstructive sLeep apnea syndrome-case report. New Med. 2015 Nov 23;19(4):130-132.

[35]. Verhulst SL, Franckx H, Van Gaal L, De Backer W, Desager K. The effect of weight loss on sleep-disordered breathing in obese teenagers. Obesity. 2009 Jun;17(6):1178-83.

[36]. Tingting, X., Danming, Y., \& Xin, C. Non-surgical treatment of obstructive sleep apnea syndrome. Eur Arch Otorhinolaryngol.2017; 275(2): 335-346.

[37]. Verma SK, Maheshwari S, Sharma NK, Prabhat KC. Role of oral health professional in pediatric obstructive sleep apnea. Natl J Maxillofac Surg. 2010 Jan;1(1):35-40.Pubmed PMID: 22442548.

[38]. de Britto Teixeira AO, Abi-Ramia LB, de Oliveira Almeida MA. Treatment of obstructive sleep apnea with oral appliances. Prog Orthod. 2013 Dec;14(1): 10

[39]. CaroleL.Marcus, LeeJay Brooks, Kari A. Draper, David Gozal, Ann Carol Halbower, Jacqueline Jones, Michael S. Schechter, Stephen Howard Sheldon, Karen Spruyt, Sally Davidson Ward, Christopher Lehmann and Richard N. Shiffman. Diagnosis and Management of Childhood Obstructive Sleep Apnea Syndrome.Pediatrics 2012;130 (3) :576-584. 